\section{POTENCIAL AGRONÓMICO DE VARIEDADES CRIOLLAS DE MAÍZ CACAHUACINTLE EN EL VALLE DE TOLUCA}

\section{AGRONOMIC POTENTIAL OF CACAHUACINTLE MAIZE LANDRACES AT TOLUCA VALLEY}

\author{
José L. Arellano Vázquez ${ }^{*}$, A. Josué Gámez \\ Vázquez ${ }^{2}$ y Miguel A. Ávila Perches²
}

\begin{abstract}
${ }^{1}$ Programa de Maíz, Campo Experimental Valle de México, Instituto Nacional de Investigaciones Forestales, Agrícolas y Pecuarias (INIFAP). Km 18.5 carretera Los Reyes-Lechería. 56230, Chapingo, Estado de México, México. Tel. 01(595)95-42877. ${ }^{2}$ Programa de Maíz, Campo Experimental Celaya. Km 6.5 carretera Celaya-San Miguel de Allende. 30018, Celaya, Guanajuato, México. Tel. 01(461)617-7107.
\end{abstract}

* Autor para correspondencia (arellano.jose@inifap.gob.mx)

\section{RESUMEN}

La producción de maíz Cacahuacintle (Zea mays L.) del Valle de Toluca se comercializa principalmente como elote y grano para pozole, a un precio de 75 a $300 \%$ por arriba del precio del maíz blanco. La producción de maíz Cacahuacintle en la región depende de las variedades criollas. En este estudio se evaluaron bajo condiciones de secano, 42 variedades criollas de maíz Cacahuacintle, y los híbridos comerciales 'H-33', 'H-44' y 'H-137', en experimentos establecidos en Calimaya y Metepec, ambas en El Valle de Toluca, Estado de México. Se utilizó el diseño experimental bloques completos al azar con tres repeticiones. Entre localidades se detectaron diferencias $(P \leq 0.01)$ para altura de planta y de mazorca, número de mazorcas por planta $y$ rendimiento de grano. Entre variedades hubo diferencias $(\mathrm{P} \leq \mathbf{0 . 0 1})$ para días a floración femenina, mazorcas por planta, acame $y$ rendimiento. Las variedades llegaron a floración femenina entre 99 y $106 \mathrm{~d}$ después de la siembra, con un nivel de acame de 12 a $24 \%$, y se caracterizaron como variedades tardías con acame de moderado a alto. El rendimiento de las mejores variedades criollas varió de 7.5 a 8.9 t $\mathrm{ha}^{-1}$ y las variedades 7,11 y 32 destacaron por sus altos valores de rendimiento, peso hectolítrico y peso de 100 semillas.

Palabras clave: Zea mays, grano harinoso, rendimiento, días a floración, tamaño del grano.

\section{SUMMARY}

The Cachuacintle maize (Zea mays L.) production in the Toluca Valley is marketed as "elote" (green and fresh ear) and as grain for "pozole", at prices 75 to $300 \%$ higher than common white grain.
Cacahuacintle maize production in the region depends on native maize landraces. In this study we evaluated 42 Cacahuacintle maize landraces and the hybrids 'H-33', 'H-44' and ' $\mathrm{H}-137$ ', in experiments established under rainfed conditions in Calimaya and Metepec, both in Toluca Valley, State of México. A randomized complete block design with three replications was used. Significant differences $(P \leq 0.01)$ were detected between localities for plant height, ear height, ears per plant and grain yield. Among landraces there were differences $(P \leq \mathbf{0 . 0 1})$ for days to silking, ears per plant, lodging percent and grain yield. Days to silking ranged from 99 to $106 \mathrm{~d}$ after sowing, and lodging from 12 to $24 \%$. These landraces were thus classified as late season varieties, with moderate to high lodging. Grain yield in the best performing landraces ranged from 7.5 to $8.9 \mathrm{t} \mathrm{ha}^{-1}$, while varieties 7,11 and 32 stood out for their high values of grain yield, hectolitric weight and weight of 100 seeds.

Index words: Zea mays, floury grain, grain yield, silking, grain size.

\section{INTRODUCCIÓN}

En México el maíz Cacahuacintle (Zea mays L.) se cultiva en los Valles Altos de la Mesa Central, en donde las áreas más importantes de producción comercial se localizan en el Valle de Toluca del Estado de México. En esa región se siembran 20000 ha para la producción de elote y 10500 ha para grano para consumo en pozole, un platillo popular en el país (González et al., 2006). Al menos $80 \%$ de la producción de maíz Cacahuacintle del Estado de México se comercializa en la Central de Abastos del Distrito Federal, como elote y grano para pozole, a un precio que supera entre 75 y $300 \%$ al precio del maíz blanco común. La producción de maíz Cacahuacintle se hace con variedades criollas nativas de la región, entre las cuales es posible identificar variedades con potencial agronómico para mejorar la productividad de este tipo de maíz.

Los objetivos del presente estudio fueron: 1) Determinar el rendimiento de grano y la expresión de caracteres de planta, mazorca y grano en variedades criollas de maíz Cacahuacintle; 2) Establecer las relaciones entre los caracteres agronómicos a través del análisis estadístico de componentes principales; y 3) Seleccionar variedades sobresalientes en función del rendimiento y caracteres de planta y grano.

\section{MATERIALES Y MÉTODOS}

Material genético. Se utilizaron 41 variedades criollas de maíz Cacahuacintle, de las cuales 25 fueron colectadas de manera directa con productores en varias regiones del Municipio de Chalchicomula, Estado de Puebla, ubicadas en altitudes de 2600 a 2900 m; las otras 16 variedades fueron colectadas en los Municipios de Calimaya, Toluca, Metepec y Tenango del Valle también del Estado de México, con altitudes similares a las localidades del Estado de Puebla. Como variedades testigo se incluyeron 
las siguientes: la Población 89 de maíz harinoso, obtenida por el Centro Internacional de Mejoramiento de Maíz y Trigo (CIMMYT), compuesta en $90 \%$ por germoplasma de la raza Cacahuacintle proveniente de los Valles Altos Mexicanos y Andinos (CIMMYT, 2001); y a los híbridos de maíz de endospermo dentado y textura semicristalina 'H-33', 'H-44' y 'H-137' obtenidos por el Instituto Nacional de Investigaciones Forestales, Agrícolas y Pecuarias (INIFAP) y que son recomendados para su siembra en áreas de temporal (o secano) o riego de los Valles Altos de la Mesa Central (Albarrán et al., 1990).

Localidades, fechas de siembra y manejo agronómico. Los experimentos se establecieron en dos localidades del Estado de México: Calimaya, ubicada a $19^{\circ} 17^{\prime} \mathrm{LN}, 99^{\circ} 39^{\prime} \mathrm{LO}$, altitud de $2675 \mathrm{~m}$, temperatura media de $12.7^{\circ} \mathrm{C}$, precipitación pluvial media de $850 \mathrm{~mm}$ y tipo de clima $\mathrm{C}(\mathrm{w} 2)(\mathrm{w}) \mathrm{big}$; y Metepec, ubicada a $19^{\circ}$ $13^{\prime} \mathrm{LN}, 99^{\circ} 35^{\prime} \mathrm{LO}$, altitud de $2606 \mathrm{~m}$, temperatura media de $13.4{ }^{\circ} \mathrm{C}$, precipitación pluvial de $890 \mathrm{~mm}$ y tipo de clima C(w2)(w)big (García, 1973). Los experimentos se desarrollaron bajo condiciones de temporal o secano, y las siembras se llevaron a cabo los días 10 y 24 de abril del 2005 en Calimaya y Metepec, respectivamente. La fertilización se hizo con la fórmula $120 \mathrm{~N}-60 \mathrm{P}-30 \mathrm{~K}$ y la densidad de población fue de 63500 plantas $\mathrm{ha}^{-1}$ (Albarrán et al., 1990).

Diseño experimental, datos registrados $\mathbf{y}$ análisis estadístico. Se utilizó el diseño experimental de bloques completos al azar con tres repeticiones, en el que el tamaño de parcela fue dos surcos de $6.0 \times 0.8 \mathrm{~m}$. Los datos registrados fueron: días a floración masculina (FLORM); días a floración femenina (FLORF); altura de planta, en $\mathrm{cm}$ (ALTPL); altura de mazorca, en $\mathrm{cm}$ (ALTMZ); acame de planta, en porcentaje (ACAME); número de mazorcas por planta (MAZPL); rendimiento de grano, en $\mathrm{t} \mathrm{ha}^{-1}$ (REND); peso hectolítrico, en $\mathrm{kg} \mathrm{hL}^{-1}$ (PHEC); y peso de 100 semillas, en $\mathrm{g}$ (P100S). Con esos datos se efectuaron análisis combinados de varianza y la prueba de Tukey al 0.05 de probabilidad del error para la comparación de medias. Además se efectuó un análisis de componentes principales. Los análisis de varianza, prueba de medias y de componentes principales se llevaron a cabo con los procedimientos indicados en SAS/STAT User's Guide (SAS Institute, 1996).

\section{RESULTADOS Y DISCUSIÓN}

Se detectaron diferencias significativas $(P \leq 0.01)$ entre localidades para los caracteres: altura de mazorca, grado de acame, número de mazorcas por planta y rendimiento (Cuadro 1). Entre variedades hubo diferencias significativas $(\mathrm{P} \leq 0.01)$ para días a floración masculina y femenina, grado de acame, número de mazorcas por planta, rendimiento, peso hectolítrico del grano y peso de 100 semillas, lo cual evidencia la variabilidad genética para esos caracteres. En la interacción variedades $\mathrm{x}$ localidades hubo diferencias $(\mathrm{P} \leq 0.01)$ para acame y rendimiento.

\section{Comparación de localidades y variedades}

En Calimaya el rendimiento fue superior $(\mathrm{P} \leq 0.01)$ en $1.2 \mathrm{t} \mathrm{ha}^{-1}$, respecto al obtenido en Metepec. Entre las variedades criollas de maíz Cacahuacintle, se detectó que 20 de ellas rindieron entre 7.5 y $8.9 \mathrm{t} \mathrm{ha}^{-1}$, lo cual representa un alto potencial de rendimiento si se considera que éstas no han sido mejoradas genéticamente, y además fueron similares al testigo ' $\mathrm{H}-44$ ' que es un híbrido triple de grano semicristalino que rindió $10.3 \mathrm{t} \mathrm{ha}^{-1}$. Las variedades llegaron a floración femenina entre los 99 y los $106 \mathrm{~d}$, con alturas de planta que fluctuaron de $250 \mathrm{a}$ $280 \mathrm{~cm}$ y con nivel de acame de 12 a $24 \%$; es decir; son variedades tardías con porte de planta muy alto y nivel de acame de moderado a alto.

En el grupo de variedades de mayor rendimiento se ubicaron tres variedades del Estado de México (32, 27, y 15) que rindieron $8.9,8.5$ y $8.4 \mathrm{t} \mathrm{ha}^{-1}$ respectivamente; estas variedades son originarias de Santa María Nativitas, Tenango del Valle y Metepec, áreas reconocidas por su alta productividad de maíz Cacahuacintle.

Cuadro 1. Cuadrados medios y significancia estadística de caracteres de planta y grano en variedades de maíz Cacahuacintle evaluadas en Calimaya y Metepec del Estado de México, 2005.

\begin{tabular}{|c|c|c|c|c|c|c|c|c|c|c|}
\hline $\begin{array}{l}\text { Fuente de } \\
\text { variación }\end{array}$ & $\mathrm{gl}$ & $\begin{array}{l}\text { FLORM } \\
\text { (d) }\end{array}$ & $\begin{array}{l}\text { FLORF } \\
\text { (d) }\end{array}$ & $\begin{array}{l}\text { ALTPL } \\
(\mathrm{cm})\end{array}$ & $\begin{array}{c}\text { ALTMZ } \\
(\mathrm{cm})\end{array}$ & $\begin{array}{c}\text { ACAME } \\
(\%)\end{array}$ & MAZPL & $\begin{array}{l}\text { REND } \\
\left(\mathrm{t} \mathrm{ha}^{-1}\right)\end{array}$ & $\begin{array}{c}\text { PHEC } \\
\left(\mathrm{kg} \mathrm{hL}^{-1}\right)\end{array}$ & $\begin{array}{l}\text { P100S } \\
(\mathrm{g})\end{array}$ \\
\hline Localidades (L) & 1 & 0.000614 & 0.00043 & 15895 & $108136^{* *}$ & $10067 * *$ & $2.6^{* *}$ & $87423180 * *$ & 1.0 & 1.2 \\
\hline Repeticiones (Loc) & 4 & 5.85 & 3.26 & 247 & 428 & $41^{* *}$ & $0.24 * *$ & $5504879 * *$ & $19.5^{*}$ & $57.2 * *$ \\
\hline Variedades (v) & 44 & $24.97 * *$ & $24.87 * *$ & 384 & 419 & $16^{*}$ & $0.08 * *$ & $4768740 * *$ & $65.6^{* *}$ & $129.4 * *$ \\
\hline $\mathrm{V} \times \mathrm{L}$ & 44 & 0.058 & 0.016 & 471 & 553 & $17 *$ & 0.03 & $2458863 * *$ & 1.15 & 1.17 \\
\hline Error total & 176 & & & & & & & & & \\
\hline $\mathrm{CV}(\%)$ & & 2.7 & 2.6 & 6.0 & 12.4 & 38.7 & 17.3 & 16.0 & 1.7 & 1.9 \\
\hline
\end{tabular}


Cuadro 2. Promedios de caracteres de planta, mazorca, grano y rendimiento, de variedades de maíz Cacahuacintle, en Calimaya y Metepec del Estado de México, 2005.

\begin{tabular}{|c|c|c|c|c|c|c|c|}
\hline Variedades & $\begin{array}{l}\text { FLORF } \\
\text { (días) }\end{array}$ & $\begin{array}{c}\text { ALTPL } \\
(\mathrm{cm})\end{array}$ & $\begin{array}{c}\text { ACAME } \\
(\%)\end{array}$ & MAZPL & $\begin{array}{l}\text { REND } \\
\left(\mathrm{t} \mathrm{ha}^{-1}\right)\end{array}$ & $\begin{array}{c}\text { PHEC } \\
\left(\mathrm{kg} \mathrm{hL}^{-1}\right)\end{array}$ & $\begin{array}{c}\text { P100S } \\
(\mathrm{g})\end{array}$ \\
\hline 44 & $105 \mathrm{a}$ & $250 \mathrm{c}$ & $12 \mathrm{~d}$ & $1.28 \mathrm{a}$ & $10.3 \mathrm{a}$ & $74 \mathrm{a}$ & $33 \mathrm{e}$ \\
\hline 32 & $101 \mathrm{~b}$ & $270 \mathrm{a}$ & $16 \mathrm{c}$ & $0.95 \mathrm{~b}$ & $8.9 \mathrm{a}$ & $64 \mathrm{~b}$ & $64 \mathrm{a}$ \\
\hline 27 & $102 \mathrm{~b}$ & $260 \mathrm{~b}$ & $19 \mathrm{~b}$ & $0.89 \mathrm{~b}$ & $8.5 \mathrm{a}$ & $59 \mathrm{~b}$ & $60 \mathrm{~b}$ \\
\hline 15 & $103 \mathrm{a}$ & $272 \mathrm{a}$ & $16 \mathrm{c}$ & $0.95 \mathrm{~b}$ & $8.4 \mathrm{a}$ & $62 \mathrm{~b}$ & $58 \mathrm{~b}$ \\
\hline 11 & $102 \mathrm{~b}$ & $274 \mathrm{a}$ & $15 \mathrm{c}$ & $0.87 \mathrm{~b}$ & $8.3 \mathrm{a}$ & $62 \mathrm{~b}$ & $58 \mathrm{~b}$ \\
\hline 7 & $103 \mathrm{a}$ & $276 \mathrm{a}$ & $18 \mathrm{~b}$ & $0.85 \mathrm{~b}$ & $8.3 \mathrm{a}$ & $61 \mathrm{~b}$ & $63 \mathrm{a}$ \\
\hline 25 & $104 \mathrm{a}$ & $269 \mathrm{a}$ & $17 \mathrm{~b}$ & $0.88 \mathrm{~b}$ & $8.2 \mathrm{a}$ & $58 \mathrm{~b}$ & $57 \mathrm{~b}$ \\
\hline 12 & $101 \mathrm{~b}$ & $269 \mathrm{a}$ & $13 \mathrm{~d}$ & $0.85 \mathrm{~b}$ & $8.1 \mathrm{a}$ & $59 \mathrm{~b}$ & $57 \mathrm{~b}$ \\
\hline 28 & $103 \mathrm{a}$ & $264 \mathrm{~b}$ & $17 \mathrm{~b}$ & $0.87 \mathrm{~b}$ & $7.9 \mathrm{a}$ & $58 \mathrm{~b}$ & $53 \mathrm{~b}$ \\
\hline 3 & $99 \mathrm{c}$ & $275 \mathrm{a}$ & $15 \mathrm{c}$ & $0.86 \mathrm{~b}$ & $7.9 \mathrm{a}$ & $62 \mathrm{~b}$ & $56 \mathrm{~b}$ \\
\hline 4 & $102 \mathrm{~b}$ & $269 \mathrm{a}$ & $16 \mathrm{c}$ & $0.81 \mathrm{~b}$ & $7.8 \mathrm{a}$ & $61 \mathrm{~b}$ & $53 \mathrm{~b}$ \\
\hline 22 & $100 \mathrm{~b}$ & $266 \mathrm{~b}$ & $19 \mathrm{~b}$ & $0.95 \mathrm{~b}$ & $7.8 \mathrm{a}$ & $60 \mathrm{~b}$ & $56 \mathrm{~b}$ \\
\hline 33 & $102 \mathrm{~b}$ & $273 a$ & $17 \mathrm{~b}$ & $0.86 \mathrm{~b}$ & $7.8 \mathrm{a}$ & $61 \mathrm{~b}$ & $66 \mathrm{a}$ \\
\hline 38 & $104 \mathrm{a}$ & $260 \mathrm{~b}$ & $16 \mathrm{c}$ & $0.95 \mathrm{~b}$ & $7.7 \mathrm{a}$ & $64 \mathrm{~b}$ & $54 \mathrm{~b}$ \\
\hline 13 & $106 \mathrm{a}$ & $280 \mathrm{a}$ & $17 \mathrm{~b}$ & $0.87 \mathrm{~b}$ & $7.7 \mathrm{a}$ & $64 \mathrm{~b}$ & $56 \mathrm{~b}$ \\
\hline 26 & $101 \mathrm{~b}$ & $257 \mathrm{~b}$ & $18 \mathrm{~b}$ & $0.95 \mathrm{~b}$ & $7.6 \mathrm{a}$ & $66 \mathrm{~b}$ & $49 \mathrm{~d}$ \\
\hline 9 & $103 \mathrm{a}$ & $262 \mathrm{~b}$ & $19 \mathrm{~b}$ & $0.88 \mathrm{~b}$ & $7.5 \mathrm{a}$ & $60 \mathrm{~b}$ & $67 \mathrm{a}$ \\
\hline 2 & $103 \mathrm{a}$ & $275 \mathrm{a}$ & $19 \mathrm{~b}$ & $0.87 \mathrm{~b}$ & $7.5 \mathrm{a}$ & $63 \mathrm{~b}$ & $49 \mathrm{~d}$ \\
\hline 1 & $103 \mathrm{a}$ & $279 a$ & $15 \mathrm{c}$ & $0.88 \mathrm{~b}$ & $7.5 \mathrm{a}$ & $62 \mathrm{~b}$ & $55 \mathrm{c}$ \\
\hline 21 & $101 \mathrm{~b}$ & $275 \mathrm{a}$ & $19 \mathrm{~b}$ & $0.84 \mathrm{~b}$ & $7.5 \mathrm{a}$ & $61 \mathrm{~b}$ & $58 \mathrm{c}$ \\
\hline 41 & $104 \mathrm{a}$ & $261 \mathrm{~b}$ & $10 \mathrm{~d}$ & $0.88 \mathrm{~b}$ & $7.5 \mathrm{a}$ & $61 \mathrm{~b}$ & $55 \mathrm{c}$ \\
\hline 34 & $104 \mathrm{a}$ & $284 \mathrm{a}$ & $23 \mathrm{a}$ & $0.85 \mathrm{~b}$ & $7.4 \mathrm{~b}$ & $60 \mathrm{~b}$ & $64 \mathrm{a}$ \\
\hline 29 & $103 \mathrm{a}$ & $276 \mathrm{a}$ & $12 \mathrm{~d}$ & $0.81 \mathrm{~b}$ & $7.3 \mathrm{~b}$ & $58 \mathrm{~b}$ & $57 \mathrm{~b}$ \\
\hline 36 & $106 \mathrm{a}$ & $284 \mathrm{a}$ & $19 \mathrm{~b}$ & $0.63 \mathrm{c}$ & $7.2 \mathrm{~b}$ & $64 \mathrm{~b}$ & $64 \mathrm{a}$ \\
\hline 42 & $101 \mathrm{~b}$ & $266 \mathrm{~b}$ & $18 \mathrm{~b}$ & $0.98 \mathrm{a}$ & $7.1 \mathrm{~b}$ & $65 \mathrm{~b}$ & $49 \mathrm{~d}$ \\
\hline 37 & $105 \mathrm{a}$ & $259 \mathrm{~b}$ & $16 \mathrm{c}$ & $0.89 \mathrm{~b}$ & $7.1 \mathrm{~b}$ & $58 \mathrm{~b}$ & $64 \mathrm{a}$ \\
\hline 6 & $102 \mathrm{~b}$ & $265 \mathrm{~b}$ & $18 \mathrm{~b}$ & $0.89 \mathrm{~b}$ & $7.0 \mathrm{~b}$ & $60 \mathrm{~b}$ & $60 \mathrm{a}$ \\
\hline 43 & $105 \mathrm{a}$ & $258 \mathrm{~b}$ & $17 \mathrm{~b}$ & $1.16 \mathrm{a}$ & $7.0 \mathrm{~b}$ & $70 \mathrm{~b}$ & $33 \mathrm{e}$ \\
\hline 39 & $103 \mathrm{a}$ & $264 \mathrm{~b}$ & $11 \mathrm{~d}$ & $0.93 \mathrm{~b}$ & $6.9 \mathrm{~b}$ & $61 \mathrm{~b}$ & $57 \mathrm{c}$ \\
\hline 20 & $102 \mathrm{~b}$ & $281 \mathrm{a}$ & $23 \mathrm{a}$ & $0.77 \mathrm{~b}$ & $6.9 \mathrm{~b}$ & $59 \mathrm{~b}$ & $57 \mathrm{c}$ \\
\hline 23 & $101 \mathrm{~b}$ & $281 \mathrm{a}$ & $24 \mathrm{a}$ & $0.69 \mathrm{c}$ & $6.9 \mathrm{~b}$ & $60 \mathrm{~b}$ & $53 \mathrm{~d}$ \\
\hline 16 & $103 \mathrm{a}$ & $255 \mathrm{~b}$ & $18 \mathrm{~b}$ & $0.79 \mathrm{~b}$ & $6.9 \mathrm{~b}$ & $58 \mathrm{~b}$ & $60 \mathrm{a}$ \\
\hline 30 & $100 \mathrm{~b}$ & $270 \mathrm{a}$ & $18 \mathrm{~b}$ & $0.87 \mathrm{~b}$ & $6.9 \mathrm{~b}$ & $63 \mathrm{~b}$ & $57 \mathrm{~b}$ \\
\hline 10 & $100 \mathrm{~b}$ & $270 \mathrm{a}$ & $16 \mathrm{c}$ & $0.75 \mathrm{~b}$ & $6.9 \mathrm{~b}$ & $59 \mathrm{~b}$ & $54 \mathrm{~b}$ \\
\hline 8 & $99 \mathrm{c}$ & $259 \mathrm{~b}$ & $24 \mathrm{a}$ & $0.74 \mathrm{~b}$ & $6.7 \mathrm{~b}$ & $61 \mathrm{~b}$ & $55 \mathrm{~b}$ \\
\hline 14 & $99 \mathrm{c}$ & $262 \mathrm{~b}$ & $17 \mathrm{~b}$ & $0.76 \mathrm{~b}$ & $6.6 \mathrm{~b}$ & $57 \mathrm{~b}$ & $56 \mathrm{~b}$ \\
\hline 31 & $104 \mathrm{a}$ & $268 \mathrm{a}$ & $13 \mathrm{~d}$ & $0.75 \mathrm{~b}$ & $6.4 \mathrm{~b}$ & $58 \mathrm{~b}$ & $54 \mathrm{~b}$ \\
\hline 35 & $106 \mathrm{a}$ & $283 \mathrm{a}$ & $19 \mathrm{~b}$ & $0.67 \mathrm{c}$ & $6.4 \mathrm{~b}$ & $57 \mathrm{~b}$ & $63 \mathrm{a}$ \\
\hline 17 & $100 \mathrm{~b}$ & $270 \mathrm{a}$ & $20 \mathrm{a}$ & $0.77 \mathrm{~b}$ & $6.4 \mathrm{~b}$ & $60 \mathrm{~b}$ & $63 \mathrm{a}$ \\
\hline 18 & $102 \mathrm{~b}$ & $260 \mathrm{~b}$ & $19 \mathrm{~b}$ & $0.69 \mathrm{~b}$ & $6.3 \mathrm{~b}$ & $59 \mathrm{~b}$ & $62 \mathrm{a}$ \\
\hline 5 & $102 \mathrm{~b}$ & $270 \mathrm{a}$ & $23 \mathrm{a}$ & $0.70 \mathrm{~b}$ & $6.2 \mathrm{~b}$ & $59 \mathrm{~b}$ & $45 \mathrm{~d}$ \\
\hline 45 & $107 \mathrm{a}$ & $270 \mathrm{a}$ & $12 \mathrm{~d}$ & $0.94 \mathrm{a}$ & $6.1 \mathrm{~b}$ & $70 \mathrm{a}$ & $36 \mathrm{e}$ \\
\hline 19 & $101 \mathrm{~b}$ & $266 \mathrm{~b}$ & $19 \mathrm{~b}$ & $0.85 \mathrm{~b}$ & $6.0 \mathrm{~b}$ & $59 \mathrm{~b}$ & $60 \mathrm{a}$ \\
\hline 24 & $99 \mathrm{c}$ & $270 \mathrm{a}$ & $21 \mathrm{a}$ & $0.77 \mathrm{~b}$ & $5.7 \mathrm{~b}$ & $62 \mathrm{~b}$ & $47 \mathrm{~d}$ \\
\hline 40 & $104 \mathrm{a}$ & $269 \mathrm{a}$ & $15 \mathrm{c}$ & $0.80 \mathrm{~b}$ & $5.3 \mathrm{~b}$ & $62 \mathrm{~b}$ & $47 \mathrm{~d}$ \\
\hline DSH 0.05 & 3.7 & 15.9 & 3.4 & 0.3 & 2.8 & 8.1 & 8.0 \\
\hline CV (\%) & 2.7 & 6.0 & 38.7 & 17.3 & 16.0 & 1.9 & 1.9 \\
\hline
\end{tabular}


Las variedades 7, 25 y 23 de Puebla rindieron 8.3, 8.2 y $7.9 \mathrm{t} \mathrm{ha}^{-1}$ respectivamente, y son originarias de San Francisco Cuautlancingo, San Antonio Atzitzintla y San Martín Ojo de Agua, respectivamente. En relación al peso hectolítrico del grano, las variedades de maíz Cacahuacintle fluctuaron entre 57 a $64 \mathrm{~kg} \mathrm{hL}^{-1}$, resultado similar al obtenido por Bonifacio et al. (2005), mientras que en el híbrido $\mathrm{H}-44$ fue de $74 \mathrm{~kg} \mathrm{hL}^{-1}$; por el contrario, en el peso de 100 semillas todas las variedades de Cacahuacintle superaron $(\mathrm{P} \leq 0.05)$ al ' $\mathrm{H}-44$ ' con valores de 45 a $64 \mathrm{~g}$ vs. $33 \mathrm{~g}$ del híbrido. Lo anterior se debe a la textura harinosa y tamaño grande del grano de Cacahuacintle, que son atributos de calidad para su consumo como pozole (Cuadro 2).

\section{Análisis de componentes principales}

De acuerdo con el análisis de componentes principales (CP, datos no mostrados), los dos primeros componentes explicaron $57 \%$ de la variación total de las variables medidas; los vectores propios asociados con la CP1 alcanzaron valores de 0.47 y 0.48 para número de mazorcas por planta y peso hectolítrico respectivamente, en tanto que para la CP2 fueron de $0.68,0.37$ y 0.31 para rendimiento, altura de planta y peso de 100 semillas, respectivamente. Tales valores son proporcionales a los coeficientes de correlación simple entre CP y las variables (Johnson y Wichern, 1999).

En la representación gráfica (Figura 1) del comportamiento de las variedades en función de CP1 y CP2, el híbrido 'H-44' se ubica en una posición aislada del resto de variedades de maíz Cacahuacintle; entre los cuadrantes I y II se ubicó un grupo de variedades criollas de Cacahuacintle con la mejor expresión de las variables involucradas en los componentes principales, es decir, las mejores variedades en función del rendimiento, caracteres de planta y grano, y que serían las de mayor potencial para la producción de maíz Cacahuacintle en la región de estudio. Entre éstas sobresalen las variedades 7, 11 y 32 originarias de San Francisco Cuautlancingo, Puebla, Ayapango y Santa María Nativitas, del Estado de México.

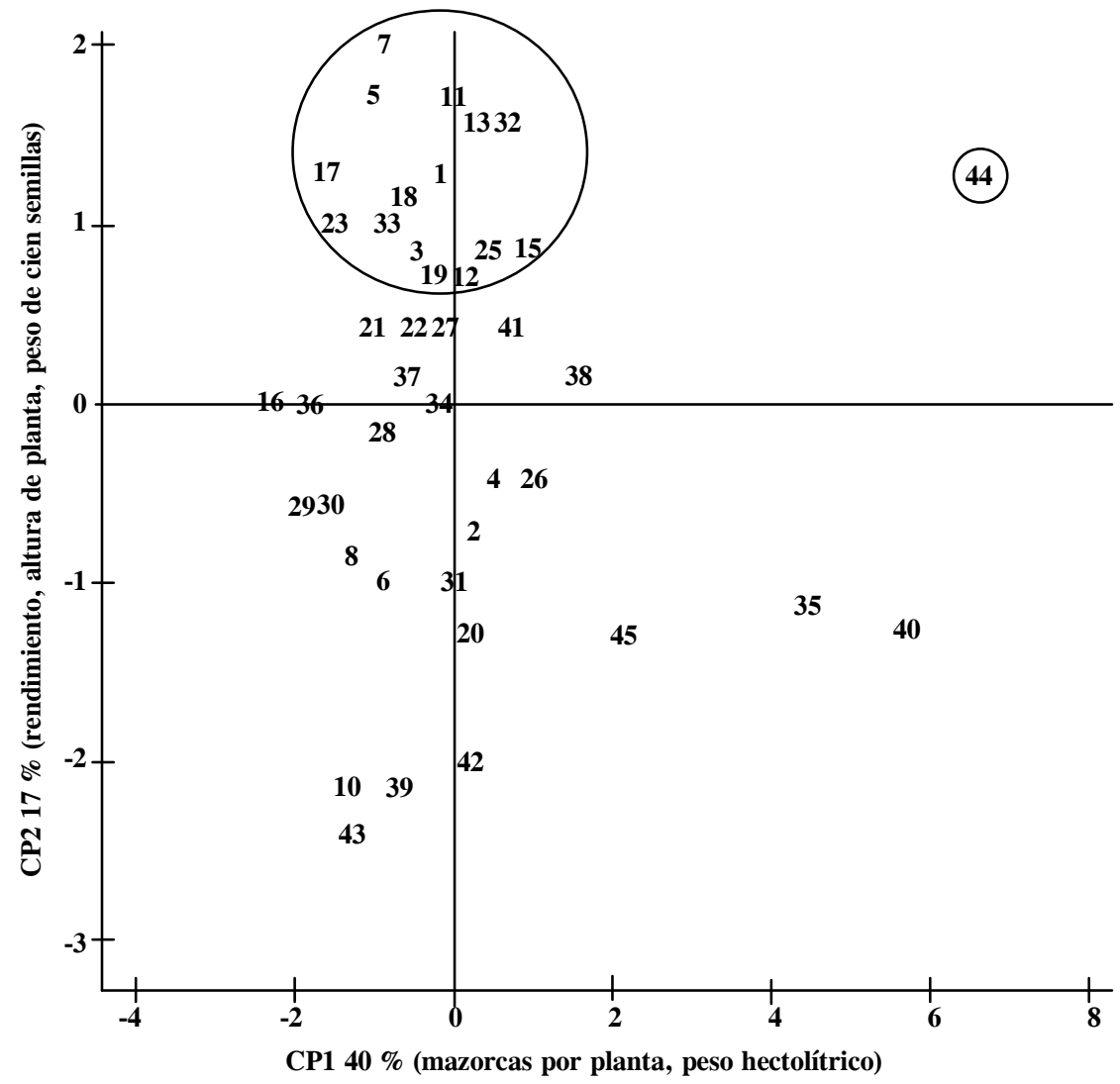

Figura 1. Representación gráfica del comportamiento de variedades criollas de maíz Cacahuacintle evaluadas en el Valle de Toluca, Estado de México. Los números representan a las variedades enlistadas en el Cuadro 2. El circulo grande agrupa a las variedades criollas sobresalientes, y el pequeño al híbrido 'H-44' (testigo). Tres variedades $(9,14$ y 24$)$ están ocultas por otras. 


\section{CONCLUSIÓN}

De las variedades criollas de maíz Cacahuacintle evaluadas, provenientes de los Estados de Puebla y México, se identificaron las sobresalientes por alto rendimiento, porte alto de planta, bajo peso hectolítrico y tamaño grande de grano, con cuya explotación se podría elevar la producción de maíz Cacahuacintle para elote y para grano "pozolero" en el Valle de Toluca.

\section{BIBLIOGRAFÍA}

Albarrán M M, J L Arellano V, R Garza G, R Valdivia B, A Espinosa C, F Alemán R, C Ortíz T, C Díaz H (1990) Guía para Cultivar Maíz en el Estado de México. Folleto para Productores Núm. 1. Secretaría de Agricultura y Recursos Hidráulicos. Instituto Nacional de Investigaciones Forestales, Agrícola y Pecuarias. Centro de Investigaciones Forestales y Agropecuarias del Estado de México. 31 p.
Bonifacio V E, Y Salinas M, A Ramos R, A Carrillo O (2005) Calidad pozolera en colectas de maíz cachuacintle. Rev. Fitotec. Mex. 28:253-260

CIMMYT, Centro Internacional de Mejoramiento de Maíz y Trigo (2001) International Maize Testing Program: 1999 Final Report. Mexico, D. F. 592 p.

García E (1973) Modificaciones al Sistema de Clasificación Climática de Köppen para Adaptarlo a las Condiciones de la República Mexicana. Instituto de Geografía. UNAM. 246 p.

González H A, J Sahagún C, D de J Pérez L, A Domínguez L, R Serrato C, V Landeros F, E Dorantes C (2006) Diversidad fenotípica de maíz cacahuacintle en el Valle de Toluca. México. Rev. Fitotec. Mex. 29: 255-261.

Johnson R A, D W Wichern (1999) Applied Multivariate Statistical Analysis. 4th ed. Prentice-Hall. Englewood Cliffs, NJ, USA $816 \mathrm{p}$.

SAS Institute (1996) SAS/STAT Guide for Personal Computers, version 6.12, SAS Institute. Cary, N. C. 1028 p. 